\title{
Involvement of $A B C$-transporters and acyltransferase 1 in intracellular cholesterol- mediated autophagy in bovine alveolar macrophages in response to the Bacillus Calmette-Guerin (BCG) infection
}

\author{
Jinrui X $\mathrm{u}^{1,2+}$, Yanbing Zhou ${ }^{1,2+}$, Yi Yang ${ }^{1,2+}$, Cuiping LV ${ }^{1,2}$, Xiaoming Liu ${ }^{1,2^{*}}$ (i) and Yujiong Wang ${ }^{1,2^{*}}$
}

\begin{abstract}
Background: Understanding pathogenic mechanisms is imperative for developing novel treatment to the tuberculosis, an important public health burden worldwide. Recent studies demonstrated that host cholesterol levels have implications in the establishment of Mycobacterium tuberculosis (M. tuberculosis, Mtb) infection in host cells, in which the intracellular cholesterol-mediated ATP-binding cassette transporters (ABC-transporters) and cholesterol acyltransferase1 (ACAT1) exhibited abilities to regulate macrophage autophagy induced by Mycobacterium bovis bacillus Calmette-Guérin (BCG).

Results: The results showed that a down-regulated expression of the ABC-transporters and ACAT1 in primary bovine alveolar macrophages (AMs) and murine RAW264.7 cells in response to a BCG infection. The inhibited expression of ABC-transporters and ACAT1 was associated with the reduction of intracellular free cholesterol, which in turn induced autophagy in macrophages upon to the Mycobacterial infection. These results strongly suggest an involvement of ABC-transporters and ACAT1 in intracellular cholesterol-mediated autophagy in AMs in response to BCG infection.
\end{abstract}

Conclusion: This study thus provides an insight into into a mechanism by which the cholesterol metabolism regulated the autophagy in macrophages in response to mycobacterial infections.

Keywords: Mycobacterium tuberculosis, Bovine, Macrophage, Cholesterol metabolism, Autophagy

\section{Background}

Tuberculosis (TB) remains a serious infectious disease worldwide, which is primarily caused by the infection of Mycobacterium tuberculosis (M. tuberculosis, Mtb), a member of the Mycobacterium tuberculosis complex (MTBC) $[1,2]$. The MTBC is a group of highly related

\footnotetext{
*Correspondence: Ixm1966@nxu.edu.cn; wyj@nxu.edu.cn

${ }^{\dagger}$ Jinrui $\mathrm{Xu}$, Yanbing Zhou and Yi Yang contributed equally to this work.

${ }^{1}$ Key Laboratory of Ministry of Education for Conservation and Utilization of Special Biological Resources in the Western, Yinchuan, China

Full list of author information is available at the end of the article
}

pathogens that are spread via an airborne route and are taken up by alveolar macrophages (AMs) in their respective hosts, of which includes bovine and human strains of the tuberculosis bacillus [3]. In this regard, Mycobacterium tuberculosis (M. tuberculosis, Mtb), Mycobacterium bovis (M. bovis) and the Mycobacterium bovis BCG vaccine strain demonstrate distinct virulence, host range and metabolism. Although, the pathogenic roles of above bacilli are expensively studied, the role of

(c) The Author(s). 2020 Open Access This article is licensed under a Creative Commons Attribution 4.0 International License, which permits use, sharing, adaptation, distribution and reproduction in any medium or format, as long as you give appropriate credit to the original author(s) and the source, provide a link to the Creative Commons licence, and indicate if changes were made. The images or other third party material in this article are included in the article's Creative Commons licence, unless indicated otherwise in a credit line to the material. If material is not included in the article's Creative Commons licence and your intended use is not permitted by statutory regulation or exceeds the permitted use, you will need to obtain permission directly from the copyright holder. To view a copy of this licence, visit http://creativecommons.org/licenses/by/4.0/ The Creative Commons Public Domain Dedication waiver (http://creativecommons.org/publicdomain/zero/1.0/) applies to the data made available in this article, unless otherwise stated in a credit line to the data. 
metabolic differences in pathogenicity remains poorly understood [4].

Autophagy is an intracellular catabolic process that helps maintain homeostasis or the removal of invading pathogens via a lysosomal degradation process [5-8]. In spite of a live attenuated vaccine against tuberculosis caused by $M t b$, the Mycobacterium bovis BCG maintains an ability to induce autophagy responses $[9,10]$, and evade phagosome maturation and autophagic degradation [11].

A compelling body of evidence has shown that the systemic cholesterol level is associated with the host immunity. Indeed, in addition to atherosclerosis and Alzheimer's disease, an abnormal cholesterol metabolism has been implicated in several lung diseases, including the development of TB [12]. Cholesterol metabolism is central to $M t b$ 's unusual ability to survive in macrophages and provide insights into potential targets for novel therapeutics $[12,13]$. In this regard, the metabolic imbalance caused by an infection of $M t b$ leads the formation of lipid droplets in macrophages, and the accumulation of lipids forms in foam cells, in order to provide a sufficient energy source for the Mycobacteria survival in host cells [14]. Recent studies in immunometabolism demonstrate the intimate link between the metabolic states of immune cells in $M t b$ infections [15], in which the host lipid metabolism is associated with the $M t b$ induced cell autophagy [16]. We sought to determine whether BCG-induced autophagy was able to be mediated by cholesterol metabolism.

It has been well documented that ATP-binding cassette transporters (ABC-transporters) [17], and cholesterol acyltransferase [18] play crucial roles in cellular cholesterol balance of immune cells such as macrophages and monocytes [19]. In this regard, ABC-transporters are a family of proteins that utilize the ATP-hydrolyzed energy to pump substrates across lipid bilayers [20]. In monocytes/macrophages, $\mathrm{ABC}$-transporters have been demonstrated to involve in reverse cholesterol transport (RCT) and forestall atherosclerotic lesion progression [21]. In this context, the PPAR $\gamma$-LXR $\alpha$-ABCA1/ABCG1 signaling is involved in regulating cholesterol efflux in macrophages, where an activation of liver X receptor alpha (LXR $\alpha$ ) directly induces the expression of the membrane ATP-binding cassette transporters, such as ABCA1 and ABCG1, which in turn pumps cholesterol out of cells [22]. Similarly, the cholesterol acyltransferases (ACATs) are exclusively intracellular enzymes that produce cholesteryl ester utilizing free cholesterols as the substrates, which is a key procession in maintaining cellular cholesterol homeostasis [23]. To date, two mammal ACATs, the ACAT1 and ACAT2 have been identified [24]. The ACAT1 is an enzyme that resides in the endoplasmic reticulum (ER) membrane of cells. It is also a major isoenzyme in macrophages [25]. An increased number of evidences showed that the ACAT1 was involved in the pathogenesis of many human diseases such as atherosclerosis, Alzheimer's disease (AD) and cancers, and it has been investigated as a potential target for treatment of these diseases [26].

With regard to bovine AMs, our previous RNA-Seq analysis revealed an alteration of $\mathrm{ABC}$-transporters and ACAT1 in BCG-infected primary bovine AMs as compared to the naïve AMs (Suppl. Fig. S1). Together with aforementioned findings, we therefore hypothesize that the BCG-altered ABC-transporters and ACAT1 may have an important implication in the regulation of intracellular cholesterol level and autophagy in macrophages in response to Mycobacterial infections. Our study will provide useful information to further unveil the function and mechanism of autophagy in mycobacterium-host interactions.

\section{Results}

Alterations of ABC-transporters and ACAT1 in BCGinfected macrophages

For further understanding the immune response macrophage induced by Mycobacterium tuberculosis infection at molecular levels, we analyzed RNA-Seq data in bovine alveolar macrophage (AM) at $12 \mathrm{~h}$ post a BCG infection. The sequencing data uncovered 1111 differential expression of mRNA between the infected group and the noninfected group, of which 426 genes were up-regulated, and 685 were down-regulated (Suppl. Fig.S1 and Table 1). Among them, the $\mathrm{ABC}$-transporters $A B C A 5$, $A B C A 6$, and $A B C A 10$ genes were down-regulated by more than 1.5 folds in primary bovine AMs infected with BCG (Table 1). Of note, the ABCA5 was reported to correlate with cholesterol efflux in macrophage, while little is known about functions of ABCA6 and ABCA10 [27], suggesting the $B C G$-altered $A B C$ transporters may have an important implication in the regulation of intracellular cholesterol in macrophages. In order to validate the RNA-Seq findings and explore the changes of other

Table 1 The differentiate expression of $A B C$ transports, $A B C A 6, A B C A 5$ and $A B C A 10$ genes in primary bovine alveolar macrophages in response to BCG infection

\begin{tabular}{llllll}
\hline Gene & BCG12_FPKM & C12_FPKM & log2 (fold change) & $P$ value & $Q$ value \\
\hline ABCA6 & 1.94886 & 5.71408 & -1.55189 & 0.001982 & 0.025436 \\
ABCA10 & 0.254202 & 3.55728 & -3.80673 & $7.05 \mathrm{E}-12$ & $1.49 \mathrm{E}-09$ \\
ABCA5 & 2.28543 & 7.0428 & -1.62368 & 0.001165 & 0.016954 \\
\hline
\end{tabular}


$\mathrm{ABC}$ transporters in macrophages, the abundance of transcripts of $A B C G 1, A B C A 1, A B C A 5$ and $A B C A 6$, as well as the acyltransferase 1 (ACAT1), a gene related to intracellular cholesterol, in both primary bovine AMs and RAW264.7 cells with BCG for $12 \mathrm{~h}$ was evaluated by a qRT-PCR assay (Suppl. Fig. S2). As expected, the expression of all above tested genes was strikingly downregulated in macrophages in response to the BCG infection (Suppl. Fig. S2). In agreement with the finding of transcripts, the down-regulated $\mathrm{ABC}$ transporters and ACAT1 was further corroborated by an immunoblotting assay, i.e. less abundant ABCG1, ABCA1, ABCA5, ABCA6 and ACAT1 proteins were observed in both BCG-infected primary bovine AMs (Fig. 1a) and murine RAW264.7 cells (Fig. 1b), in comparison with the naïve macrophages. Of interest, the least ABCA1 and ACAT1 proteins in BCG-infected bovine AMs were observed at post infection $6 \mathrm{~h}$, while the ABCG1 was at $12 \mathrm{~h}$, and the least $\mathrm{ABCA} 5$ and $\mathrm{ABCA} 6$ were at $24 \mathrm{~h}$ post infection (Fig. 1a). Accordingly, the least ABCG1, ABCA1 and
ACAT1 proteins in BCG-infected RAW264.7 cells were found at $12 \mathrm{~h}$ post infection, while the least ABCA5 and ABCA6 were at $24 \mathrm{~h}$ post infection (Fig. 1b).

\section{The BCG infection increases intracellular cholesterol in macrophages}

The ABC-transporters ABCG1, ABCA1, ABCA5 participate in the reverse cholesterol transport from macrophages and ACAT1 converts free cholesterol to cholesteryl esters [28]. A down regulation of these proteins may imply an increase of intracellular cholesterol. Indeed, a dynamic change of intracellular cholesterol and cholesterol ester with time was observed in both bovine AMs and macrophages in response to the BCG infection (Fig. 2). In this context, intracellular levels of free cholesterol (Fig. 2a) and cholesterol ester (Fig. 2b) of primary bovine AMs were gradually increased and decreased, respectively. The intracellular free cholesterol (Fig. 2a) and cholesterol ester (Fig. 2b) reached their peaks at $6 \mathrm{~h}$ post infection before they
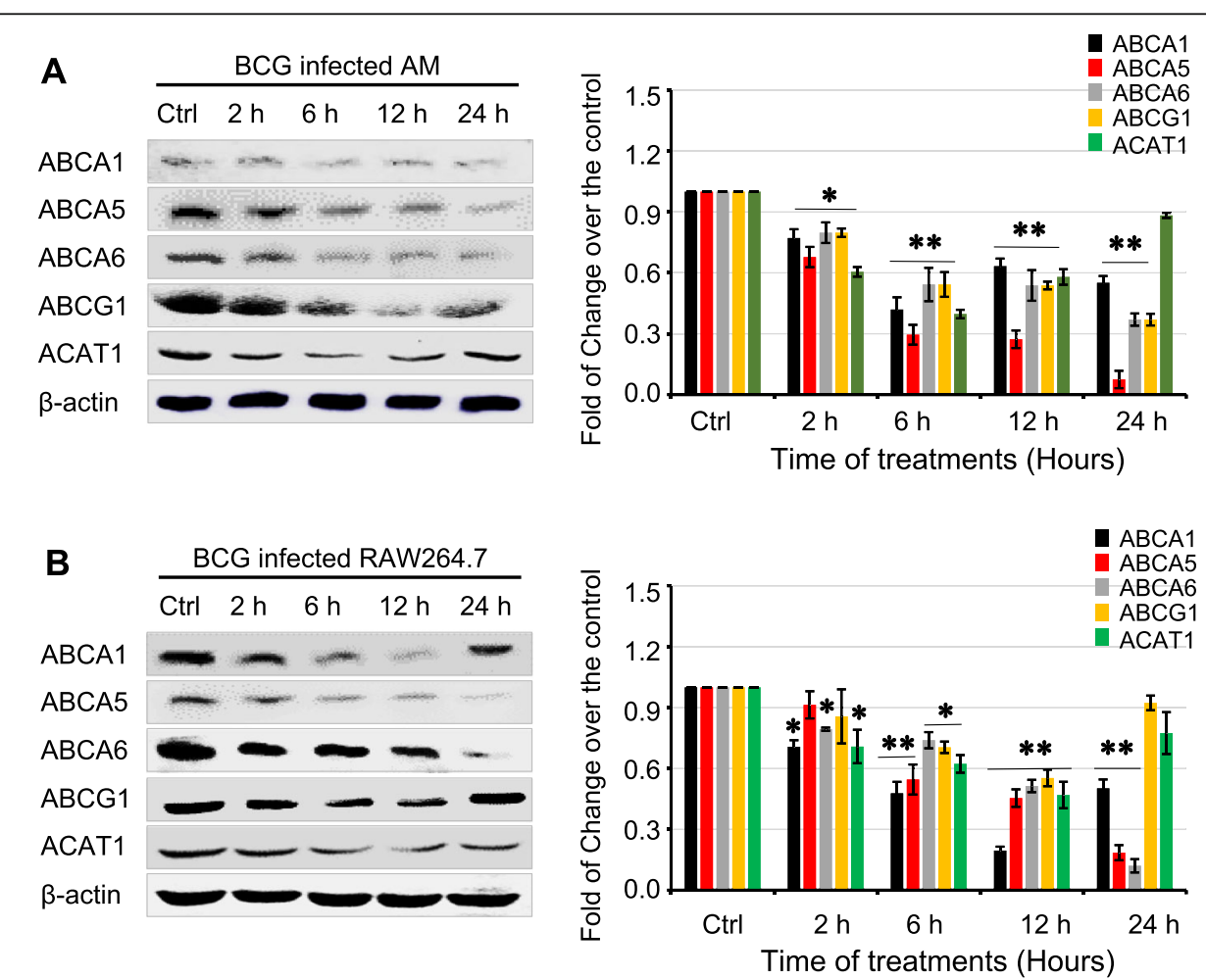

Fig. 1 The dynamic changes of the expression of ABC-transporters and ACAT1 in macrophages response to a BCG infection. Primary bovine alveolar macrophages (AMs) and murine macrophage RAW264.7 cells were infection with BCG at a dose of 10 for indicated time periods, abundances of ABC-transporters and ACAT1 were determined by an immunoblotting (IB) assay. a Representative images of blots for indicated proteins of interest of BCG-infected primary bovine AMs probed with corresponding antibodies (Left panel), and the relative expression of proteins semi-quantified by an optical densitometry analysis (Right panel) demonstrated a dynamic change of protein expression (b) Representative images of blots for indicated proteins of interest of BCG-infected murine macrophage RAW264.7 cells probed with corresponding antibodies (Left panel), and the relative expression of proteins semi-quantified by an optical densitometry analysis (Right panel) demonstrated a dynamic change of protein expression. Both the BCG-infected bovine AMs and RAW264.7 cells exhibited the lowest expression of ABCA1, ACAT1 $A B C G 1, A B C A 5$ and $A B C A 6$ at 6, 6, 12, 24 and $24 \mathrm{~h}$ post the BCG infection, respectively. Data were expressed as mean \pm SEM from three independent experiments. Compared to non-infection controls, ${ }^{*}: p<0.05 ;{ }^{* *}: p<0.01$ 

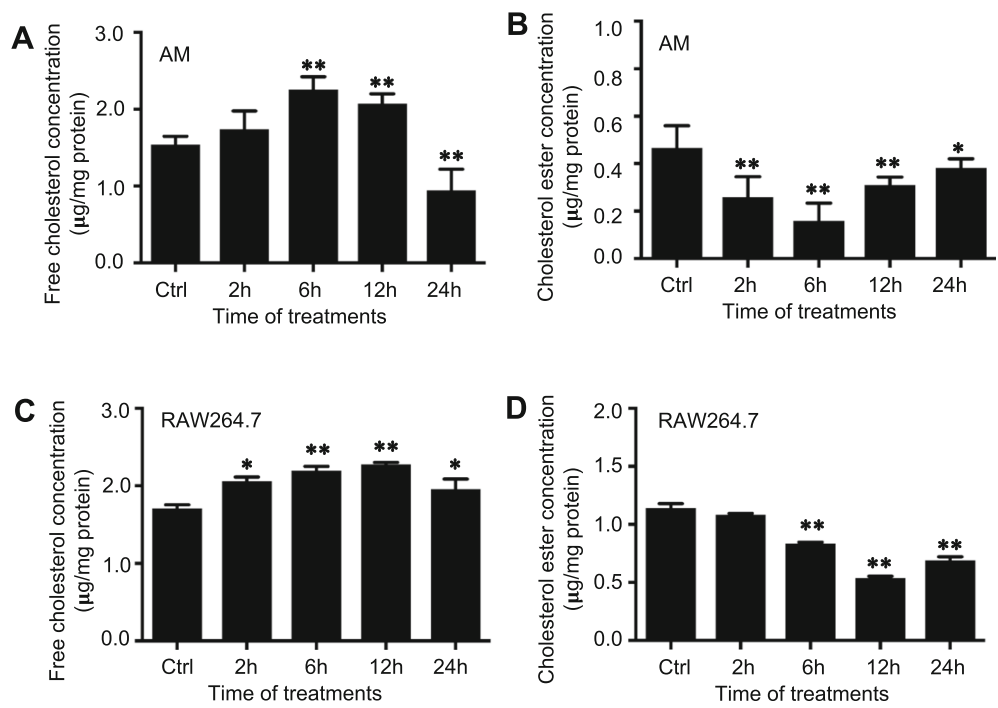

Fig. 2 The BCG infection altered intracellular levels of cholesterol and cholesterol ester in macrophages. Bovine AMs and murine macrophage RAW264.7 cells were infected with BCG at a dose of 10 for indicated times before their intracellular levels of cholesterol and cholesterol ester were ascertained. $\mathbf{a}-\mathbf{b}$ Intracellular levels of cholesterol (a) and cholesterol ester (b) of primary bovine AMs altered by the infection of BCG. $\mathbf{a}$ The content of intracellular free cholesterol was increased gradually and reached its peak at $6 \mathrm{~h}$ post infection before it significantly decreased from $12 \mathrm{~h}$ afterward; $\mathbf{b}$ while the intracellular cholesterol ester content in AMs was significantly decreased and reached the lowest level at $6 \mathrm{~h}$ and then dramatically increased at 12 and afterward. c-d Intracellular levels of cholesterol (c) and cholesterol ester (d) of murine RAW264.7 cells altered by BCG. $\mathbf{c}$ The intracellular free cholesterol was increased and reached its peak at $12 \mathrm{~h}$ but reduced at $24 \mathrm{~h}$ post infection. A significant change of intracellular cholesterol was observed between $2 \mathrm{~h}, 6 \mathrm{~h}$ and $12 \mathrm{~h}$ after infection as compared with the control group; in contrast, $\mathbf{d}$ the intracellular cholesterol ester was significantly decreased and reached the lowest level at $12 \mathrm{~h}$ but increased at $24 \mathrm{~h}$ post infection. A significant change of intracellular cholesterol ester was determined between cells at $6 \mathrm{~h}, 12 \mathrm{~h}$ and $24 \mathrm{~h}$ following the infection, as compared with the control group. Data were expressed as mean \pm SEM from three independent experiments. Compared to non-infection control, * $p<0.05,{ }^{* *}: p<0.01$

respectively decreased and increased from $12 \mathrm{~h}$ afterward. Consistently, the dynamic changes of intracellular free cholesterol (Fig. 2c) and cholesterol ester (Fig. 2d) were also detected in BCG-infected RAW264.7 cells. The intracellular free cholesterol was increased and reached its peak at $12 \mathrm{~h}$ but reduced at $24 \mathrm{~h}$ post infection (Fig. 2c); while the intracellular cholesterol ester was significantly decreased and reached the lowest level at $12 \mathrm{~h}$ but increased at $24 \mathrm{~h}$ post infection (Fig. 2d). These results may indicate that the increased intracellular cholesterol is a consequence of BCGinhibited expression of $\mathrm{ABC}$-transporters and ACAT1.

\section{Infection of BCG induces autophagy in macrophages}

Accumulating evidences have demonstrated that autophagy can be induced by cholesterol in Mycobacteriainfected macrophages [29]. In line with other studies, a significant increased expression of autophagy markers LC3II/I and Beclin1 was observed in primary bovine AMs (Fig. 3a) and RAW264.7 cells (Fig. 3b). Interestingly, the BCG-induced expression of autophagy markers showed a comparably dynamic trend with the change of intracellular cholesterol in both cell types (Fig. 2), indicating that the intracellular cholesterol may play a role in regulating autophagy in BCG-infected macrophages, which needs further investigation.
The involvement of ACAT1-mediated intracellular cholesterol in autophagy of macrophages in response to BCG infection

The ACAT1 gene has been demonstrated to correlate with intracellular cholesterol and autophagy [26]. In order to further validate the involvement of the alteration of ACAT1 in BCG-infected macrophages, RAW264.7 stable cell lines overexpressing and silencing ACAT1 were generated by lentiviral vector-mediated gene transduction (data not shown). As expected, the overexpression of ACAT1 significantly decreased the BCG-induced intracellular free cholesterol, while the silence of ACAT1 expression led an increased BCG-induced intracellular free cholesterol (Fig. 4a). In consistence, an overexpression of ACAT1 restored the BCG-inhibited intracellular cholesterol ester, while a silence of ACAT1 aggravated the suppression of BCG-reduced intracellular cholesterol ester (Fig. 4b). Of importance, the ACAT1-altered intracellular cholesterol and cholesterol ester were correlated with the abundance of protein markers of autophagy in macrophages infected with BCG (Fig. 5). An overexpression ACAT1 reduced the BCG-induced expression of autophagy-related proteins ATG5, ATG7 LC3II/I and Beclin1 (Fig. 5a), while knocking-down of ACAT1 expression enhanced the BCGinduced autophagy proteins in RAW264.7 cells (Fig. 5b). This funding was further corroborated by an experiment 

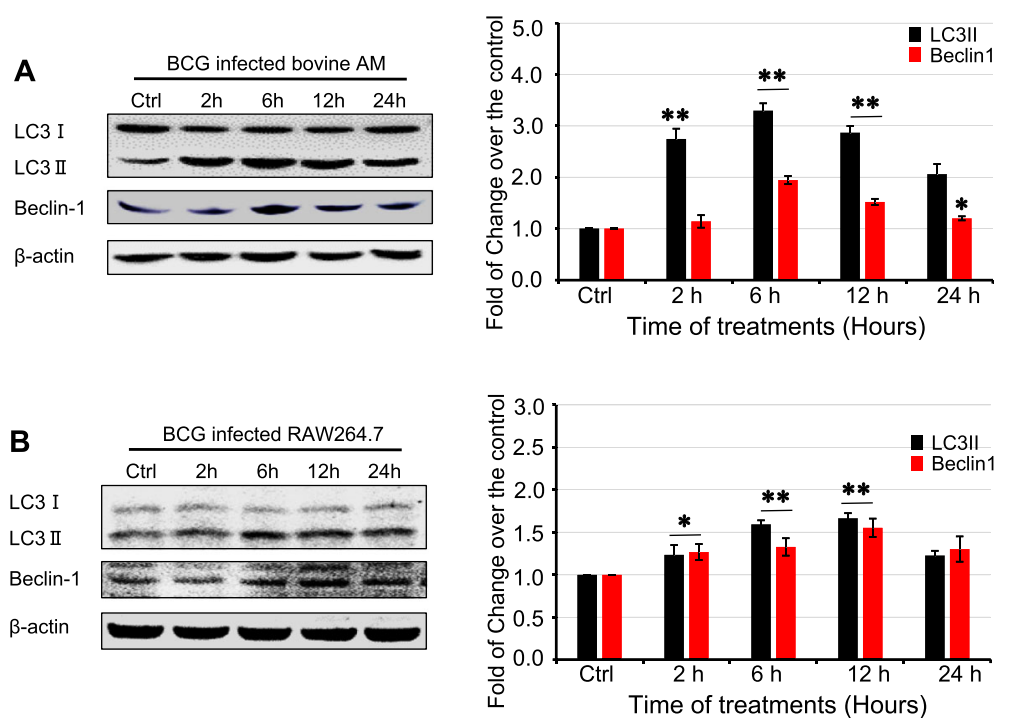

Fig. 3 BCG infection induced the expression of autophagy-related proteins in macrophages. Bovine AMs and murine macrophage RAW264.7 cells were infected with BCG at a dose of 10 for indicated times, and the expression of autophagy-related proteins LC3I/II and Beclin 1 was determined by an immunoblotting (IB) assay. a Representative images of blots for LC3//II and Beclin1 in BCG-infected primary bovine AMs (left panel), and the relative expression of proteins semi-quantified by a densitometry analysis (right panel) demonstrated an increased expression of autophagyrelated proteins. b Representative images of blots of LC31/II and Beclin1 in BCG-infected murine macrophage RAW264.7 cells (left panel), and their relative expression semi-quantified by a densitometry analysis (right panel) indicated an induced autophagy by BCG infection. Data were expressed as mean \pm SEM from three independent experiments. Compared to non-infection controls, ${ }^{*}: p<0.05 ;{ }^{* *}: p<0.01$

using ACAT1 specific inhibitor K604 (Fig. 6). In the presence of K604, the BCG-induced autophagy-related proteins ATG5, ATG7, LC3II/I and Beclin1 were significantly increased in both primary bovine AMs (Fig. 6a) and murine RAW264.7 cells (Fig. 6b), as compared with their respective controls in the absence of K604. These results strongly suggest an involvement of ACAT1 in regulation of intracellular cholesterol and autophagy of macrophages in response to BCG Infection.

\section{Discussion}

Both of bovine and human strains of the tuberculosis bacillus, Mtb and Bovis tuberculosis are members of MTBC. Despite they demonstrate distinct difference in virulence, host range and metabolism, the metabolic differences of these bacilli in pathogenicity remains exclusive [4]. The ability of the pathogen $M t b$ to metabolize steroids like cholesterol plays a key role in the virulence and pathogenesis [30]. Cholesterol, a major structural
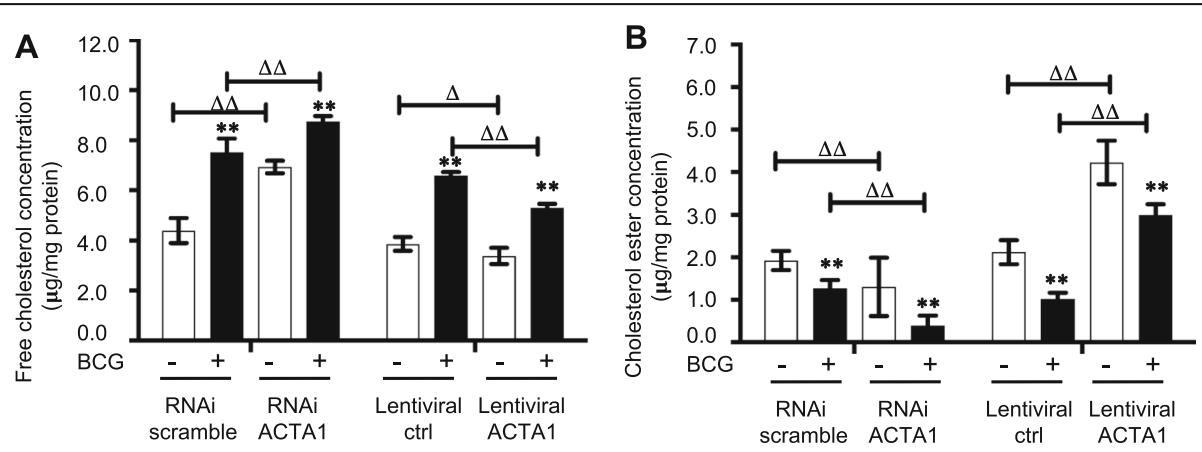

Fig. 4 An ACAT1-mediated alteration of intracelluar cholesterol and cholesterol ester in macrophages in response to BCG infection. Murine RAW264.7 cell lines overexpressing and silencing ACAT1 were generated by lentiviral infections with vectors overexpressing ACAT1 or shRNA to ACAT1, respectively. Control RAW264.7 cell lines infected with appropriate control lentiviral vectors were also generated. The transgenic RAW264.7 cells were infected with BCG at a dose of 10 for $12 \mathrm{~h}$ prior to being harvested for assessment of intracellular cholesterol (a) and cholesterol ester (b). a An overexpression of ACAT1 exhibited an ability to significantly suppress BCG-induced intracellular cholesterol, while knockdown of ACAT1 showed an enhanced level of intracellular cholesterol in RAW264.7 cells. b An overexpression of ACAT1 significantly restored BCG-repressed intracellular cholesterol ester, while knockdown of ACAT1 further reduced the BCG-repressed cholesterol ester in RAW264.7 cells. Data were expressed as mean \pm SEM from three independent experiments. Compared to non-BCG-infected cells, $*^{*} p<0.05$; **: $p<0.01$; compared to noncontrol lentivirus-infected cells, ${ }^{\Delta}: p<0.05 ;{ }^{\Delta \Delta}: p<0.01$ 


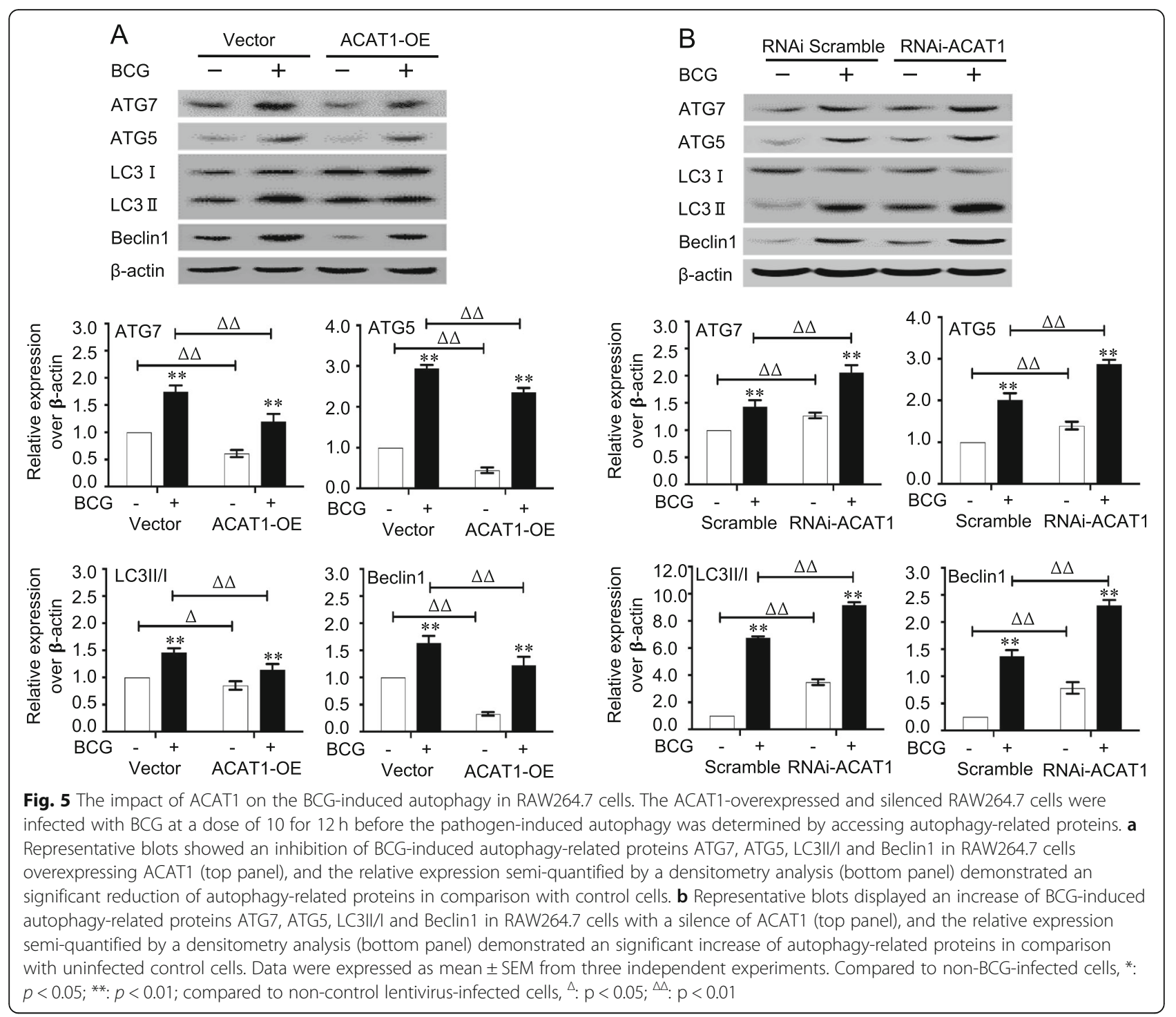

component of animal cell membranes, is thought to be involved in immune regulations [31] and the development of $M t b$ infection [30]. In this regard, $M t b$ is able to import and metabolize host cholesterol during infection, which is critical for the maintenance of $M t b$ infection [30]. In addition, cholesterol also is a critical carbon source during latent $M t b$ infection, during which $M t b$ is capable of using cholesterol as a carbon source [32]. Moreover, the cholesterol of macrophage host has been shown to facilitate the entry of mycobacteria into macrophages [33], where mycobacteria-activated macrophages effectively transfer the phagocytosed pathogens to the destructive microenvironment of lysosomes [34]. As an important component of mammalian cells with many basic cellular functions, the intracellular cholesterol level is regulated many factors such as Acyl-CoA: cholesterol acyltransferase 1 (ACAT1) and ABC transporters [35]. Functionally, the ACAT1 is the major isoenzyme in macrophages [25], which converts cholesterol to cholesterol esters, and plays important roles in lipoprotein assembly, dietary cholesterol absorption, and intracellular cholesterol metabolism [36].

In this study, a down-regulated ATP-banding transporters family members related to cholesterol efflux, were observed in BCG-infected bovine AMs by RNA-seq analysis. The results suggested that BCG infectionaltered the intracellular cholesterol was regulated by $\mathrm{ABC}$ transporter-mediated cholesterol efflux. This hypothesise was supported by alterations of the expression of ABCA5, ABCA6, ABCA1, ABCG1 at both transcriptional and translational levels in $\mathrm{AMs}$ and murine RAW264.7 cells. On the other hand, the expression of Acyl-CoA: cholesterol acyltransferase1 (ACAT1) was also altered in these cells, suggesting its important role in regulating the intracellular cholesterol in macrophages in response to mycobacterial infections. 


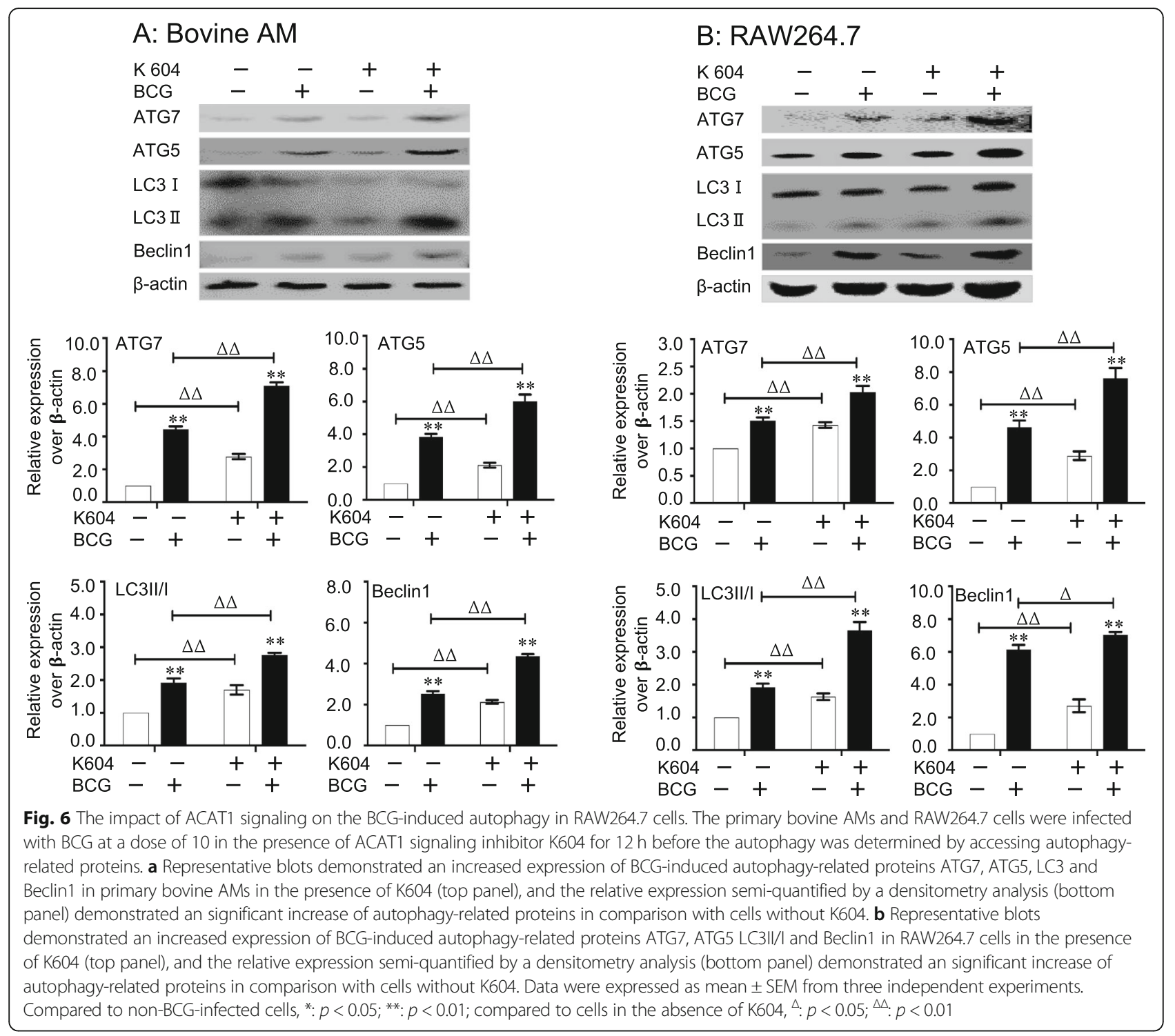

Programmed cell death and autophagy are fundamental processes of cell biology intimately involved in the interaction between Mycobacteria and their infected phagocytes [37]. In this regard, autophagy can target $M t b$ and promote phagosomal maturation to inhibit the replication of intracellular bacilli. However, the bacilli have an ability to escape from lysosome and survive within their host macrophages by utilizing host resources as early niches for their replication and growth in cells. Emerging evidence suggests that the cholesterol metabolism plays a crucial role in the regulation of macrophage autophagy [31, 38]. In line with these findings, we also observed a trend of that the change of cholesterol in macrophages was consistent with the expression of autophagy-related proteins during BCG infection. Functionally, the ACAT1 is the major isoenzyme in macrophages [24], which plays important roles in lipoprotein assembly, dietary cholesterol absorption, and intracellular cholesterol metabolism [31]. This notion was further corroborated in AMs and RAW264.7 cells by targeting ACAT1 by altering its expression and/or introducing small molecule inhibitor K604.

Indeed, an overexpression or silence of ACAT1 in murine RAW264.7 cells led a respectively decreased and increased intracellular ACAT1 intracellular free cholesterol, but an opposite effect in intracellular cholesterol ester in response to BCG infections. Interestingly, the ACAT1-mediated alterations of intracellular cholesterol were positively correlated with the BCG-induced autophagy in macrophages. Of note, the ACAT1 inhibitor K604 exhibited a similar effect to shRNA-mediated ACAT1 knockdown in RAW264.7 cells, suggesting an involvement of ACAT1-regulated intracellular cholesterol in BCG-induced autophagy. In addition to the ACAT1, ABC transporters, ABCA1 and ABCG1 showed 
an ability to pump cholesterol out of macrophages [22]. In addition, we also found that $\mathrm{ABC}$-transporters were involved in the alteration of intracellular cholesterol in in both bovine alveolar macrophages and murine RAW264.7 macrophages infected with BCG. These data thus suggest a regulatory role of efflux transporters of macrophages in maintaining intracellular cholesterol and cell autophagy during BCG infection.

\section{Conclusions}

In summary, in the present study, we explored the regulatory roles of ABC-transporters and ACAT1 in cholesterol metabolism of bovine macrophages in response to an infection of Mycobacterium bovis vaccine stain BCG. Our results demonstrated that the infection of BCG inhibited the expression of $\mathrm{ABC}$-transporters and ACAT1 in primary bovine AMs and murine RAW264.7 cells, which in turn reduced the intracellular free cholesterol and increased cholesterol ester. Importantly, the BCG-inhibited expression of ABC-transporters and ACAT1, and its consequently reduced intracellular cholesterol was correlated with the BCG-induced autophagy in macrophages, clearly indicating an involvement of $\mathrm{ABC}$-transporters and ACAT1 in intracellular cholesterol-mediated autophagy in macrophages in response to Mycobacterial infections. This study thus highlights an importance of metabolism of intracellular cholesterol in macrophages, aids our better understanding in the innate immune mechanisms during mycobacterial infection and eventually provides useful information for development of host-directed therapy (HDT) strategies in TB treatments.

\section{Methods}

Primary bovine alveolar macrophages and RAW264.7 cells This study was approved by the ethics committee for use and care of animals at Ningxia University (Yinchuan, China). Primary bovine AMs were obtained from lungs of one- to two-year-old Simendal cattles bred in a tuberculosis-free herd. The entire lung was sterilely removed post-mortem with a portion of trachea, and was intratracheally infused with $500 \mathrm{~mL}$ of D-Hank's solution (Biotopped, Beijing, China) containing $50 \mu \mathrm{g} / \mathrm{mL}$ of gentamicin, $2.5 \mu \mathrm{g} / \mathrm{mL}$ of amphotericin and $100 \mu \mathrm{g} / \mathrm{mL}$ of penicillin and streptomycin (Hyclone, Logan, USA). The recovered bronchoalveolar lavage fluid (BALF) was filtered by passing a $70 \mu \mathrm{m}$-pore nylon cell strainer prior to being collected into sterile beakers and centrifuged at $1000 \mathrm{rpm}$ for $10 \mathrm{~min}$. The cell pellet was then resuspended in $20 \mathrm{~mL}$ RPMI-1640 medium (HyClone, Logan, USA) supplemented with 10\% Fetal Bovine Serum (FBS, Gibco, Carlsbad, USA), $100 \mathrm{U} / \mathrm{mL}$ of penicillin and 100 $\mathrm{U} / \mathrm{mL}$ of streptomycin. $3 \mathrm{~mL}$ of red blood cell (RBC) lysate solution was then added into the cell suspension and gently mixed well for $3 \mathrm{~min}$ at room temperature (RT) before the cells were recollected by centrifugation at $1000 \mathrm{rpm}$ for $10 \mathrm{~min}$. The cells were resuspended with RPMI-1640 medium with 10\% FBS and counted. The resultant primary cells were seeded at a density of $5 \times 10^{7}$ per tissue culture dish with diameter of $140 \mathrm{~mm}$ and cultured in RPMI-1640 medium with 10\% FBS for 6 h to 8 $h$. The unattached cells were removed by rinsing the culture with pre-warmed PBS, and the attached monolayer of primary AMs were dissociated with Tryple ${ }^{\mathrm{TM}}$ Express (Thermo Fisher Scientific, Shanghai, China) and harvested by centrifugation. The isolated cells were then replated in 6-well plates at a density of $5 \times 10^{6} /$ well in RPMI-1640 medium with 10\% FBS and cultured for $16-$ $18 \mathrm{~h}$ for subsequent experiments. The murine macrophage cell line RAW264.7 was purchased from Shanghai Academy of Life Sciences, Chinese Academy of Sciences (Shanghai, China). Cells were maintained in DMEM medium supplemented with $10 \%$ calf serum and penicillin/streptomycin. All cells were cultured in humidified incubators with $5 \% \mathrm{CO}_{2}$ atmosphere at $37^{\circ} \mathrm{C}$.

\section{BCG culture and infection}

The $M$. bovis $B C G$ vaccine strain was purchased from Chengdu Institute of Biological products (Chengdu, China). BCG bacterial cells were grown in the Middlebrook 7H9 medium (BD Difco, San Jose, CA, USA) supplemented with $10 \%$ albumin-dextrose-catalase (ADC) enrichment medium (BD Difco, San Jose, CA, USA) and 0.05\% Tween 80 (Sigma, St. Louis, MO, USA) at $37^{\circ} \mathrm{C}$ with slow shaking for 2 weeks. The bacteria cells were harvested by centrifugation and re-suspended in the culture medium. The bacterial cell number was titrated by spectrophotometer at wavelength of $600 \mathrm{~nm}$ based on an OD600nm of 1.0 equivalent to $1 \times 10^{8}$ mycobacterial cells [39]. The bacteria stocks were aliquot and stored at $-80{ }^{\circ} \mathrm{C}$ freezer for subsequent uses. For infection, the macrophage cells were cultured with 6-well plates for 6$8 \mathrm{~h}$ before they were infected with BCG at a multiplicity of infection (MOI) of 10 bacteria and then incubated for additionally various times.

\section{RNA-seq analysis}

The sequencing of transcriptome was performed by Beijing Novogene Bioinformatics Technology Company. The sequencing platform was Illumina Hi SeqTM4000. TopHat2 was used to conduct comparative analysis of the reference genome of filtered reads [40]. Each sample of the species was quantitatively analyzed using HTSeq software. SNP Calling and InDel Calling were respectively conducted by the mutation detection software GATK2 [41]. mRNA, lncRNA and TUCP transcript were for quantitatively analyzed using Cuffdiff (http://coletrapnell-lab.github.io/cufflinks/cuffdiff/index.html) software. Differential expression was carried out using 
Cuffdiff v2.1.1. Functional classification using the GOSeq Release 2.12 with gene ontology (GO) were carried out on genes that were found to be differentially expressed with an adjusted $p$-value of $<0.05$ [41].

\section{Quantitative RT-PCR}

The total RNA of cells was isolated using Trizol reagent per manufacturer's instruction (Invitrogen, Grand Island, NY, USA). The reverse transcription of first-strand cDNA synthesis was generated using M-MLV reverse transcriptase (TaKaRa, Dalian, China). The quantitative reverse transcription PCR (qRT-PCR) was performed in the Roche Lightcycler 2.0 using TaKaRa SYBR Green I kit (Takara, Dalian, China). The primer sets used for RT-PCR were designed and synthesized in Shanghai Sangon Biotech Inc. (Shanghai, China) by bioinformatics tools using available mRNA sequences (Suppl. Table 1). The relative expression of genes of interest was calculated by accessing the efficiencies and the crossing point deviation of a given gene vs housekeeping $\beta$-actin gene. In each independent experiment, The relative gene expression was represented by the fold of change over its respective uninfected control cultures by a $2^{-\Delta \Delta \mathrm{CT}}$ method.

\section{Immunoblotting analysis}

Whole cell extracts were prepared by lysing cell cultures in lysis buffer $(50 \mathrm{mM}$ Tris-HCl, pH 7.5, 5 mM EDTA, 150 $\mathrm{mM} \mathrm{NaCl}, 0.5 \% \mathrm{NP}-40$ ) for $60 \mathrm{~min}$ on ice. The concentration of soluble protein was determined with Bio-Rad Protein Assay based on the method of Bradford (Bio-Rad Laboratories, Richmond, CA, USA). The clarified lysates $(100 \mu \mathrm{g})$ were resolved in $8 \%$ or $10 \%$ sodium dodecyl sulfate (SDS)-polyacrylamide gel (SDS-PAGE) and then transferred to nitrocellulose membranes for immunoblotting assay probed with antibodies to proteins of interest. The primary antibodies used in this study were listed in Suppl. Table 2. All these primary antibodies were applied in a dilution of 1:500-1000. Following extensive washing, protein of interest was detected or visualized with an appropriate HRP-labelled or fluorescence-labelled IRDye (Li-Cor Biosciences, Lincoln, NE, USA) secondary antibody. The blots were then developed using the enhanced Western Bright ECL reagent (Advansta, Menlo Park, CA, United States) or Li-Cor Odassay Scanner (Li-Cor Biosciences). The relative expression of protein was semi-quantified by optical densitometry using ImageJ Software version 1.46 (http://rsb.info. nih.gov/ij/). The densitometric arbitrary unit (A.U.) was used for determined the ratio between the net intensity by calculating values of each sample divided by the $\beta$-actin internal control.

\section{Determination of cholesterol and cholesterol ester}

The contents of intracellular cholesterol and cholesterol ester were determined by commercially available cholesterol quantitative assay kit according to manufacturer's instructions (Sigma, St. Louis, MO, USA). The absorbance was then read at $570 \mathrm{~nm}$. Finally, the contents of intracellular total cholesterol and free cholesterol were calculated comparing to its standard curve, and the amount of cholesterol ester was calculated by the substrate of the content of total cholesterol to the content of free cholesterol.

\section{Generation of RAW264.7 cell lines overexpressing or silencing ACAT1}

To generate a lentiviral vector overexpressing ACAT1, cDNA of murine acat1 gene (NM_144784) was cloned downstream of the CMV promoter of GV492 lentiviral proviral backbone plasmid (Genechem Co., Ltd., Shanghai, China); to generate lentiviral vectors knocking down endogenous ACAT1 expression, shRNAs targeting sequences of 5'TCGGTCTGGCTAGTATTTG3', 5'CGTACCTAA GGTTCTTAAA3' and 5'TAACTGATGTCTACAATAA3' of murine acat1 gene (NM_144784) were respectively cloned downstream of the U6 promoter of GV493 lentiviral proviral backbone plasmid (Genechem Co.,Ltd., Shanghai, China). The constructed proviral plasmids were used for generation of respective VSV-G pseudotyped lentiviral vectors Lenti-ACAT1 and Lenti-shRNA-ACAT1 as described elsewhere. To generate murine macrophage RAW264.7 cells overexpressing and silencing ACAT1, RAW264.7 cells were infected with Lenti-ACAT1 and Lenti-shRNAACAT1, respectively. The virally transduced cells were then cultured for $72 \mathrm{~h}$ following the infection before they were refreshed with a selective medium containing purinomycin for additional 4-5 days. The cell pools with were then used for further experiments after functional determination.

\section{Suppression of ACAT1 using inhibitor K604}

RAW264.7 cells were seeded in a 6-well plate culture dish at a density of $2 \times 10^{6} /$ well in DMEM-10\% FBS culture medium containing $10 \mu \mathrm{M}$ of ACAT1 protein inhibitor K604 (MedChemExpress, USA) and BCG at a multiplicity of infection of 10:1. The cells were cultured for $12 \mathrm{~h}$ before they were used for analysis.

\section{Statistical analysis}

All data collected in this study were from at least three independent biological repeated experiments, which were analysed using SPSS statistics 22.0 (SPSS Inc., Chicago, IL, USA). The data were presented as the mean \pm standard derivation (SD). Statistical differences between groups were analysed by one-way analysis of variance (ANOVA), followed by post-hoc Tu-key's test. The data were presented as the mean \pm SD. $P$ values $<0.05$ were considered as statistically significant. 


\section{Supplementary information}

Supplementary information accompanies this paper at https://doi.org/10. 1186/s12865-020-00356-x.

Additional file 1: Supplemental file 1 Figure S1. The difference of expression primary bovine alveolar macrophages (AMs) in response to of BCG infections. The volcano plots showed the difference in the expression of genes in primary bovine alveolar macrophage (AM) infected with BCG at $12 \mathrm{~h}$ at a dose of 10 were analyzed RNA-Seq. The results revealed that there were 1111 differentially expressed genes between the infected group and the non-infected group, of which 426 were up-regulated, and 685 were down-regulated. Figure S2. The BCG infection suppresses the expression of ABC-transporters and ACAT1 in bovine alveolar macrophages (AMs). Bovine AMs were infected with BCG at $\mathrm{MOI}$ of 10 for $12 \mathrm{~h}$, and the transcripts of ABC-transporters and ACAT1 was assessed by a qRT-PCR assay. (A-E) Inductions of indicated transcripts of bovine alveolar macrophages (AMs) infected with BCG. (A) Fold of changes of ABCA1 transcript over the non-infected cells; (B) Fold of changes of ABCA5 transcript over the non-infected cells; (C) Fold of changes of ABCA6 transcript over the non-infected cells; (D) Fold of changes of ABCG1 transcript over the non-infected cells; (E) Fold of changes of ACAT1 transcript over the non-infected cells. Data represent the mean \pm the standard error of the mean (SEM) from three independent experiments. Compared to non-infection control, ${ }^{*}: p<0.05,{ }^{* *}: p<$ 0.01. Suppl. Table 1. Primer sets of qRT-PCR used in this study.

\section{Abbreviations}

ABCA1: ATP binding cassette subfamily A member 1; ABCA5: ATP binding cassette subfamily A member 5; ABCA6: ATP binding cassette subfamily A member 6; ABCA10: ATP binding cassette subfamily A member 10; ABCG1: ATP-binding cassette subfamily G member 1; ABC-transporters: ATPbinding cassette transporters; ACAT: Acyl coenzyme A:cholesterol acyltransferase; ACAT1: Acyl coenzyme A:cholesterol acyltransferase 1; ACAT2: Acyl coenzyme A:cholesterol acyltransferase 2; AD: Alzheimer's disease; AM: Alveolar macrophages; ATG5: Autophagy-Related Protein 5; ATG7: Autophagy-Related Protein 7; BCG: Bacillus Calmette-Guerin; DC: Dendritic cells; ER: Endoplasmic reticulum; HDT: Host-directed therapy; LC3: Microtubule associated protein light chain 3; LXRa: Liver X receptor alpha; M. bovis: Mycobacterium bovis; MOI: Multiplicity of infection; Mtb: Mycobacterium tuberculosis; qRT-PCR: The quantitative reverse transcription PCR; RCT: Reverse cholesterol transport; TB: Tuberculosis

\section{Acknowledgements}

The authors thank Mr. Fuyang Song for technical assistance, and other lab members for valuable discussions and assistance.

\section{Authors' contributions}

$Y W$ and $X L$ conceived and designed the experiments; JX, YY, YZ, and $C L$ performed the experiments and acquired data, $Z Y B$ and $Y Y$ analyzed the data; LCP and XJR drafted the manuscript; YW and XL interpreted data and critically revised the manuscript. All authors read and approved the final version of the manuscript.

\section{Funding}

This work was supported by Program of National Nature Science Foundation of China (Grant No 31772710, No 31760733, No 31960700, No 31960712) and key research and development program of Ningxia (2017BN04) and a grant from Natural Science Foundation of Ningxia (2019AAC03010). These funding play no role in the design of the study collection, analysis, and interpretation of data and in writing the manuscript.

\section{Availability of data and materials}

The datasets used and/or analysed during the current study are available from the corresponding author on reasonable request.

\section{Ethics approval and consent to participate}

The experiments involving bovie were performed according to protocols approved by the Institutional Animal Care and Use Committee of Ningxia University (NXU-2017-069). The need for consent is deemed unnecessary according to national regulations, but an informed verbal consent was obtained from the farmers. The ethics committee for the use of animals of Ningxia University approved this study.

\section{Consent for publication}

Not applicable.

\section{Competing interests}

The authors declare that they have no conflicts of interest.

\section{Author details}

${ }^{1}$ Key Laboratory of Ministry of Education for Conservation and Utilization of Special Biological Resources in the Western, Yinchuan, China. ${ }^{2}$ College of Life Science, Ningxia University, Yinchuan 750021, Ningxia, China.

Received: 28 August 2019 Accepted: 4 May 2020

Published online: 12 May 2020

\section{References}

1. Walzl G, McNerney R, du Plessis N, Bates M, McHugh TD, Chegou NN, Zumla A. Tuberculosis: advances and challenges in development of new diagnostics and biomarkers. Lancet Infect Dis. 2018;18(7):e199-210.

2. Paik S, Kim JK, Chung C, Jo EK. Autophagy: a new strategy for host-directed therapy of tuberculosis. Virulence. 2019;10(1):448-59.

3. Vegh P, Magee DA, Nalpas NC, Bryan K, McCabe MS, Browne JA, Conlon KM Gordon SV, Bradley DG, MacHugh DE, et al. MicroRNA profiling of the bovine alveolar macrophage response to Mycobacterium bovis infection suggests pathogen survival is enhanced by microRNA regulation of endocytosis and lysosome trafficking. Tuberculosis. 2015;95(1):60-7.

4. Lofthouse EK, Wheeler PR, Beste DJ, Khatri BL, Wu H, Mendum TA, Kierzek AM, McFadden J. Systems-based approaches to probing metabolic variation within the Mycobacterium tuberculosis complex. PLoS One. 2013;8(9): e75913.

5. Deretic V, Saitoh T, Akira S. Autophagy in infection, inflammation and immunity. Nat Rev Immunol. 2013;13(10):722-37.

6. Wang $K$, Chen $Y$, Zhang $P$, Lin $P$, Xie N, Wu M. Protective Features of Autophagy in Pulmonary Infection and Inflammatory Diseases. Cells. 2019;8(2):E123.

7. $\quad$ Ding S, Qu Y, Yang S, Zhao Y, Xu G. Novel miR-1958 promotes Mycobacterium tuberculosis survival in RAW264.7 cells by inhibiting autophagy via Atg5. J Microbiol Biotechnol. 2019;29(6):989-98.

8. Liang M, Habib Z, Sakamoto K, Chen X, Cao G. Mycobacteria and autophagy: many questions and few answers. Curr Issues Mol Biol. 2017;21:63-72.

9. Khan A, Bakhru P, Saikolappan S, Das K, Soudani E, Singh CR, Estrella JL, Zhang D, Pasare C, Ma Y, et al. An autophagy-inducing and TLR-2 activating BCG vaccine induces a robust protection against tuberculosis in mice. NPJ Vaccines. 2019:4:34.

10. Wu X, Zhang J, Ma C, Li W, Zeng J, Wang Y, Deng G. A role for Wnt/betacatenin signalling in suppressing Bacillus Calmette-Guerin-induced macrophage autophagy. Microb Pathog. 2019;127:277-87.

11. Zullo AJ, Lee S. Mycobacterial induction of autophagy varies by species and occurs independently of mammalian target of rapamycin inhibition. J Biol Chem. 2012;287(16):12668-78.

12. Van der Geize R, Yam K, Heuser T, Wilbrink MH, Hara H, Anderton MC, Sim E, Dijkhuizen L, Davies JE, Mohn WW, et al. A gene cluster encoding cholesterol catabolism in a soil actinomycete provides insight into Mycobacterium tuberculosis survival in macrophages. Proc Natl Acad Sci U S A. 2007;104(6):1947-52

13. Savvi S, Warner DF, Kana BD, McKinney JD, Mizrahi V, Dawes SS. Functional characterization of a vitamin B12-dependent methylmalonyl pathway in Mycobacterium tuberculosis: implications for propionate metabolism during growth on fatty acids. J Bacteriol. 2008;190(11):3886-95.

14. Sallese A, Suzuki T, McCarthy C, Bridges J, Filuta A, Arumugam P, Shima K, Ma $Y$, Wessendarp M, Black D, et al. Targeting cholesterol homeostasis in lung diseases. Sci Rep. 2017;7(1):10211.

15. Shi L, Jiang Q, Bushkin Y, Subbian S, Tyagi S. Biphasic Dynamics of Macrophage Immunometabolism during Mycobacterium tuberculosis Infection. MBio. 2019:10(2):e02550-18.

16. Quigley J, Hughitt VK, Velikovsky CA, Mariuzza RA, El-Sayed NM, Briken V. The Cell Wall Lipid PDIM Contributes to Phagosomal Escape and Host Cell Exit of Mycobacterium tuberculosis. MBio. 2017;8(2):e00148-17. 
17. Fessler MB. A New Frontier in Immunometabolism. Cholesterol in Lung Health and Disease. Ann Am Thorac Soc. 2017;14(Supplement_5):S399-405.

18. Chistiakov DA, Bobryshev YV, Orekhov AN. Macrophage-mediated cholesterol handling in atherosclerosis. J Cell Mol Med. 2016;20(1):17-28.

19. Genoula M, Marin Franco JL, Dupont M, Kviatcovsky D, Milillo A, Schierloh P, Morana EJ, Poggi S, Palmero D, Mata-Espinosa D, et al. Formation of foamy macrophages by tuberculous pleural effusions is triggered by the Interleukin-10/signal transducer and activator of transcription 3 Axis through ACAT upregulation. Front Immunol. 2018;9:459.

20. Davis $\mathbf{W}$ Jr. The ATP-binding cassette transporter-2 (ABCA2) regulates esterification of plasma membrane cholesterol by modulation of sphingolipid metabolism. Biochim Biophys Acta. 2014;1841(1):168-79.

21. Spartano NL, Lamon-Fava S, Matthan NR, Ronxhi J, Greenberg AS, Obin MS, Lichtenstein AH. Regulation of ATP-binding cassette transporters and cholesterol efflux by glucose in primary human monocytes and murine bone marrow-derived macrophages. Exp Clin Endocrinol. 2014; 122(8):463-8.

22. Wang $H$, Yang $Y$, Sun $X$, Tian F, Guo S, Wang W, Tian Z, Jin H, Zhang Z, Tian $Y$. Sonodynamic therapy-induced foam cells apoptosis activates the phagocytic PPARgamma-LXRalpha-ABCA1/ABCG1 pathway and promotes cholesterol efflux in advanced plaque. Theranostics. 2018;8(18):4969-84.

23. Huang LH, Melton EM, Li H, Sohn P, Rogers MA, Mulligan-Kehoe MJ, Fiering SN, Hickey WF, Chang CC, Chang TY. Myeloid acyl-CoA:cholesterol acyltransferase 1 deficiency reduces lesion macrophage content and suppresses atherosclerosis progression. J Biol Chem. 2016;291(12):6232-44.

24. Song BL, Wang CH, Yao XM, Yang L, Zhang WJ, Wang ZZ, Zhao XN, Yang JB, Qi W, Yang XY, et al. Human acyl-CoA:cholesterol acyltransferase 2 gene expression in intestinal Caco-2 cells and in hepatocellular carcinoma. Biochem J. 2006;394(Pt 3):617-26.

25. Sakashita N, Miyazaki A, Chang CCY, Chang TY, Kiyota E, Satoh M, Komohara Y, Morganelli PM, Horiuchi S, Takeya M. Acyl-coenzyme a : cholesterol acyltransferase 2 (ACAT2) is induced in monocyte-derived macrophages: in vivo and in vitro studies. Lab Investig. 2003;83(11):1569-81.

26. Shibuya Y, Chang CC, Chang TY. ACAT1/SOAT1 as a therapeutic target for Alzheimer's disease. Future Med Chem. 2015;7(18):2451-67.

27. Wang R, Sheps JA, Ling V. ABC transporters, bile acids, and inflammatory stress in liver cancer. Curr Pharm Biotechnol. 2011;12(4):636-46.

28. Hutchins PM, Heinecke JW. Cholesterol efflux capacity, macrophage reverse cholesterol transport and cardioprotective HDL. Curr Opin Lipidol. 2015; 26(5):388-93.

29. Larrouy-Maumus G. Cholesterol acquisition by Mycobacterium tuberculosis. Virulence. 2015;6(5):412-3.

30. Wipperman MF, Yang M, Thomas ST, Sampson NS. Shrinking the FadE proteome of Mycobacterium tuberculosis: insights into cholesterol metabolism through identification of an alpha2beta2 heterotetrameric acyl coenzyme a dehydrogenase family. J Bacteriol. 2013;195(19):4331-41.

31. Suzuki A, Shim J, Ogata K, Yoshioka H, Iwata J. Cholesterol metabolism plays a crucial role in the regulation of autophagy for cell differentiation of granular convoluted tubules in male mouse submandibular glands. Development. 2019;146(20):dev178335.

32. Abuhammad A. Cholesterol metabolism: a potential therapeutic target in mycobacteria. Br J Pharmacol. 2017;174(14):2194-208.

33. Kumar GA, Jafurulla M, Chattopadhyay A. The membrane as the gatekeeper of infection: cholesterol in host-pathogen interaction. Chem Phys Lipids. 2016;199:179-85.

34. VanderVen BC, Fahey RJ, Lee W, Liu Y, Abramovitch RB, Memmott C, Crowe AM, Eltis LD, Perola E, Deininger DD, et al. Novel inhibitors of cholesterol degradation in Mycobacterium tuberculosis reveal how the bacterium's metabolism is constrained by the intracellular environment. PLoS Pathog. 2015;11(2):e1004679.

35. Fessler MB. The intracellular cholesterol landscape: dynamic integrator of the immune response. Trends Immunol. 2016;37(12):819-30

36. Buhman KF, Accad M, Farese RV. Mammalian acyl-CoA:cholesterol acyltransferases. Biochim Biophys Acta. 2000;1529(1-3):142-54.

37. Moraco AH, Kornfeld $\mathrm{H}$. Cell death and autophagy in tuberculosis. Semin Immunol. 2014;26(6):497-511.

38. Barbero-Camps E, Roca-Agujetas V, Bartolessis I, de Dios C, Fernandez-Checa JC, Mari M, Morales A, Hartmann T, Colell A. Cholesterol impairs autophagymediated clearance of amyloid beta while promoting its secretion. Autophagy. 2018;14(7):1129-54.
39. Penuelas-Urquides K, Villarreal-Trevino L, Silva-Ramirez B, RivadeneyraEspinoza L, Said-Fernandez S, de Leon MB. Measuring of Mycobacterium tuberculosis growth. A correlation of the optical measurements with colony forming units. Braz J Microbiol. 2013;44(1):287-9.

40. Kim D, Pertea G, Trapnell C, Pimentel H, Kelley R, Salzberg SL. TopHat2: accurate alignment of transcriptomes in the presence of insertions, deletions and gene fusions. Genome Biol. 2013;14(4):R36.

41. McKenna A, Hanna M, Banks E, Sivachenko A, Cibulskis K, Kernytsky A, Garimella K, Altshuler D, Gabriel S, Daly M, et al. The genome analysis toolkit: a MapReduce framework for analyzing next-generation DNA sequencing data. Genome Res. 2010;20(9):1297-303.

\section{Publisher's Note}

Springer Nature remains neutral with regard to jurisdictional claims in published maps and institutional affiliations.

\section{Ready to submit your research? Choose BMC and benefit from:}

- fast, convenient online submission

- thorough peer review by experienced researchers in your field

- rapid publication on acceptance

- support for research data, including large and complex data types

- gold Open Access which fosters wider collaboration and increased citations

- maximum visibility for your research: over $100 \mathrm{M}$ website views per year

At BMC, research is always in progress.

Learn more biomedcentral.com/submissions 